\title{
Transient neonatal diabetes and later onset diabetes: a case of inherited insulin resistance
}

\author{
J P H Shield, J D Baum
}

\begin{abstract}
A 13 year old girl who had had transient neonatal diabetes developed permanent diabetes. She had raised fasting insulin concentrations suggestive of insulin resistance with a suboptimal insulin response to glucose loading. Both her mother and sister had profound insulin resistance; neither had clinical diabetes. This is the first time inherited insulin resistance has been implicated in the pathogenesis of permanent diabetes developing after transient neonatal diabetes.

(Arch Dis Child 1995; 72: 56-57)
\end{abstract}

Keywords: transient neonatal diabetes, insulin resistance.

Transient neonatal diabetes mellitus (TNDM) classically occurs in small for dates, term infants. They develop hyperglycaemia, severe dehydration but minimal ketosis, requiring insulin treatment, within the first six postnatal weeks. The natural history of the condition has been considered benign with the infants becoming euglycaemic without insulin treatment by 1 year of age. ${ }^{1}$ A satisfactory explanation for TNDM has never been established. However, 'pancreatic dysmaturity' leading to reduced insulin production has been proposed, ${ }^{2}$ as has insulin resistance, ${ }^{3}$ and most recently insulin imprinting in neonatal life. ${ }^{4}$ Until recently it was felt that there were no long term sequelae. This view has recently been challenged with nine cases (including this child), from the 60 cases of TNDM in the literature, now known to have developed diabetes in later life. In the two reported cases of later onset diabetes undergoing dynamic tests, hypoinsulinaemia with no evidence of autoimmunity or genetic susceptibility to type 1 diabetes has been found. 56

\section{Case report}

A term, small for dates $(1700 \mathrm{~g},<3$ rd centile) girl was born to non-diabetic parents. Twelve hours after delivery she developed hyperglycaemia with severe dehydration but minimal ketosis. Minimal insulin treatment $(0.5 \mathrm{U} / \mathrm{kg} / \mathrm{day})$ was required for the first three postnatal months. At approximately 3 months of age, a dynamic test of glucose loading was reported to show undetectable insulin concentrations. Mild abnormalities in glucose handling and insulin production remained until the age of 6 months. Once insulin independent, she remained well until 13 years of age when diabetes mellitus was diagnosed with hyperglycaemia, dehydration, and mild ketosis responsive to insulin treatment. The child requires minimal insulin treatment $(0.25$ $\mathrm{U} / \mathrm{kg} /$ day) to maintain excellent blood glucose control with glycated haemoglobin concentrations nearly always within the non-diabetic range. Subsequent to our recent investigations the patient, when aged 16 , was managed by diet alone for the period of one month with no exogenous insulin treatment and no significant deterioration in clinical condition or blood glucose control. The child's maternal grandfather had developed non-insulin dependent diabetes aged 59 years. There was no other family history of diabetes.

\section{Investigations}

INDEX CASE

A standard oral glucose tolerance test (OGTT; $1.75 \mathrm{~g} / \mathrm{kg}$ up to a maximum of $75 \mathrm{~g}$ ) when the patient was aged 15 years, performed after discontinuing insulin treatment from the day before testing, showed a raised fasting insulin concentration of $192 \mathrm{pmol} / \mathrm{l}$ (normal range
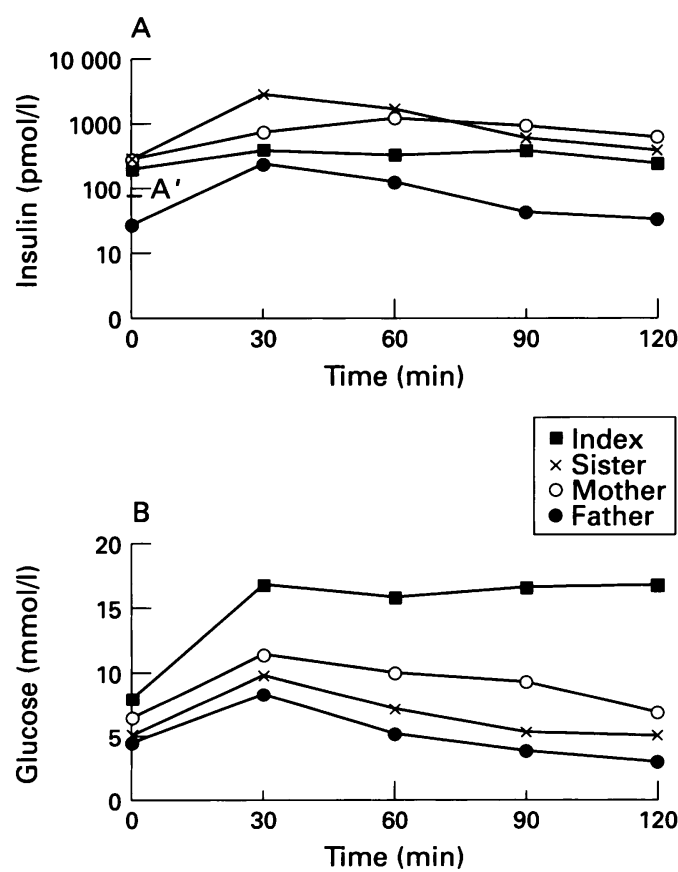

(A) Plasma insulin (on $\log _{10}$ axis) and (B) plasma glucose responses to standard oral glucose loading of the entire family. The father's responses are included for normal comparison. Upper limit for normal fasting insulin 60 pmoll $\left(A^{\prime}\right)$. The World Health Organisation definition for normal plasma glucose values in the adult during oral glucose tolerance test are fasting $<6.4 \mathrm{mmol}$ and at two hours $<7.8 \mathrm{mmol}$. For children the upper normal range are fasting $<7.2 \mathrm{mmol} / \mathrm{l}$ and at two hours $<7.8 \mathrm{mmol}$. Diabetes mellitus in adult or child is defined at fasting $>7.7 \mathrm{mmol} /$ and at two hours $>11.0 \mathrm{mmol}$. 
30-60) with a blunted maximum response to glucose loading of $385 \mathrm{pmol} / 1$ and a frankly diabetic glucose curve (figure). HLA typing revealed homozygous A3, B27, DR2 (16), DQ6. Investigation for specific islet cell autoantibody production was negative. The child was non-obese with a body mass index of 23.5. Fasting lipid concentrations were normal and blood pressure was $112 / 49 \mathrm{~mm} \mathrm{Hg}$.

FAMILY

As the initial diagnosis of classical childhood onset diabetes at 13 years of age seemed questionable, family studies of glucose tolerance were undertaken. An OGTT performed on the 18 year old non-diabetic, mildly obese sister (body mass index 28 ) revealed an even higher concentration of fasting insulin of $237 \mathrm{pmol} / 1$ and an exaggerated insulin response to glucose loading with a maximum at 30 minutes of $3024 \mathrm{pmol} / \mathrm{l}$ (figure). Fasting lipid concentrations were again normal. Blood pressure was $122 / 62 \mathrm{~mm} \mathrm{Hg}$. The sister's birth weight had been normal at $3400 \mathrm{~g}$.

OGTT on the non-diabetic obese mother (body mass index 44 ) showed a similar picture with raised fasting insulin concentrations of $244 \mathrm{pmol} / \mathrm{l}$ and a maximum response to glucose loading of $1156 \mathrm{pmol} / \mathrm{l}$ (figure). Fasting triglyceride was raised at $2.5 \mathrm{mmol} / \mathrm{l}$ $(0 \cdot 5-1 \cdot 7)$ and cholesterol slightly raised at $6 \cdot 2$ $\mathrm{mmol} / 1$ (optimal $<5 \cdot 2$ ). Her blood pressure was $148 / 108 \mathrm{~mm} \mathrm{Hg}$. Her birth weight had been normal at $3800 \mathrm{~g}$. The father's OGTT was entirely normal (figure).

\section{Discussion}

This child developed childhood onset diabetes 13 years after TNDM; other children with similar biographies have recently been reported. ${ }^{5}$ As in our case, a number have been shown to carry the DR2 haplotype that is usually protective against childhood onset insulin dependent diabetes. This, together with the absence of islet cell antibodies, suggests that these children have an aetiopathogenesis unlike classical childhood onset diabetes. We report the first case of TNDM with later onset permanent diabetes in whom there is evidence of a familial insulin resistance syndrome. We speculate that intrauterine growth retardation was associated with suboptimal pancreatic development predisposing the child to long term abnormalities in insulin production that in the face of an insulin resistance syndrome led to the re-emergence of diabetes. By way of contrast, both her sister and mother, who were of normal birth weight, are able to produce the exceptionally high concentrations of insulin needed to maintain normoglycaemia in response to a glucose challenge.

We hypothesise, from the number of reports of permanent diabetes developing after TNDM, that the endocrine capability of the pancreas in these subjects is permanently affected. Additionally, our case indicates that other factors such as insulin resistance may influence the later development of clinical diabetes.

We would like to thank Dr S O'Rahilly of the departments of medicine and clinical biochemistry, University of Cambridge, for his assistance with the laboratory analysis of insulin concentrations.

1 Gentz JCH, Cornblath M. Transient diabetes of the newborn. Adv Pediatr 1969; 16: 345-63.

2 Milner RDG, Ferguson AW, Naidu SH. Aetiology of transient neonatal diabetes. Arch Dis Child 1971; 46: 724-6.

3 Chance GW, Bower BD. Hypoglycaemia and temporary hyperglycaemia in infants of low birth weight for maturity. Arch Dis Child 1966; 41: 279-85.

4 Haig D. Is human insulin imprinted? Nature Genetics 1994; 7: 10.

5 Weimerskirch D, Klein DJ. Recurrence of insulin dependent diabetes mellitus after transient neonatal diabetes: a report of two cases. F Pediatr 1993; 122: 598-600.

6 Gottschalk ME, Schatz DA, Clare-Salzer M, Kaufman DL Ting GSP, Geefner ME. Permanent diabetes without serological evidence of autoimmunity after transient neonatal diabetes. Diabetes Care 1992; 15: 1273-6. 